\title{
Minimizing the Diagnosis of "Follicular Lesion of Undetermined Significance" and Identifying Predictive Features for Neoplasia
}

Xin Jing, M.D., ${ }^{1 *}$ Michael H. Roh, M.D., Ph.D., ${ }^{1}$ Stewart M. Knoepp, M.D., Ph.D., ${ }^{1}$ Lili Zhao, Ph.D., ${ }^{2}$ and Claire W. Michael, M.D. ${ }^{1}$

We used proposed standard morphologic criteria as a guideline to conduct a 10-year retrospective review of thyroid fine-needle aspiration specimens that were originally interpreted as "follicular lesion of undetermined significance" and followed by surgical intervention. We sought to investigate whether the indeterminate diagnosis could be minimized by assessing various cytomorphologic features and identifying the features predictive of neoplasia. Using the standard morphologic criteria, we semiquantitatively assessed a total of 24 cytomorphologic features in 123 aspirates and recorded an overall interpretation on completion of the review. Cyto-histologic correlation was evaluated and logistic regression model was performed to identify cytomorphologic features predictive of neoplasia. Although 32 of 123 aspirates remained in the indeterminate category, the retrospective review reclassified 64 aspirates as non-neoplasia and 27 aspirates as neoplasia. Histologic confirmation was achieved in 47 (73.4\%) non-neoplastic and 15 (55.6\%) neoplastic aspirates with a diagnostic accuracy of $68.1 \%$. Furthermore, our analysis demonstrated that neoplasia is positively associated with the presence of syncytial tissue fragments, isolated microfollicles, follicles with scalloped borders, scant cytoplasm, irregular nuclear membranes, nuclear overlapping, coarse chromatin, and increased cellularity. On the contrary, the presence of honeycombing tissue fragments, background colloid, and histiocytes inversely correlated with neoplasia.

Overall, using proposed standard morphological criteria can minimize the diagnosis of "follicular lesion of undetermined significance," and allow for more accurate cyto-histologic correla-

\footnotetext{
${ }^{1}$ Department of Pathology, University of Michigan, Ann Arbor, Michigan

${ }^{2}$ Biostatistics Unit, University of Michigan, Ann Arbor, Michigan

Grant sponsor: University of Michigan, Department of Pathology Anatomic Pathology Project Fund.

*Correspondence to: Xin Jing, M.D., Department of Pathology, University of Michigan, 1500 E. Medical Center Drive, 2G332 UH, Ann Arbor, MI 48109-0054. E-mail: xinjing@med.umich.edu

Received 10 February 2010; Accepted 1 May 2010

DOI 10.1002/dc.21459

Published online 14 October 2010 in Wiley Online Library (wileyonlinelibrary.com).
}

tion, and thereby playing a substantial role in reducing unnecessary surgical intervention. Diagn. Cytopathol. 2011;39:737742. 02010 Wiley-Liss, Inc.

Key Words: FNA; thyroid nodules; follicular lesion of undetermined significance

Fine-needle aspiration (FNA) of thyroid is an effective triage method in the management of thyroid nodules. It aims to distinguish neoplastic nodules that require surgical intervention from non-neoplastic nodules that may be conservatively managed by clinical and radiologic followup. ${ }^{1-4}$ It is well known that a straightforward cytologic diagnosis may not be rendered for every aspirate partially due to inherent cytological similarities between lesions. ${ }^{5-7}$ The recent National Cancer Institute (NCI) Thyroid FNA State of the Science Conference recommended use of the category "follicular lesion of undetermined significance" for thyroid aspirates in which cytomorphologic findings are neither convincingly benign nor sufficient for interpretation of neoplasia/malignancy. ${ }^{8}$ Previous studies claimed that the use of this category reduced the false-negative rate of $\mathrm{FNA}^{6}$ while eliminating this category substantially increased both false-positive and false-negative rates. ${ }^{9}$ Judicious use of this diagnostic category is encouraged and follow-up with repeat aspiration and correlation with clinical and radiologic findings is suggested by the committee members of the NCI conference. ${ }^{8}$

FNA of thyroid nodules has been a growing practice within our institution with 600-800 thyroid FNAs being evaluated annually in recent years. Nearly $6 \%$ were classified as "follicular lesion of undetermined significance." Although this indeterminate interpretation represents a minority of the total FNA diagnoses, the predictive uncertainty for malignancy, inherent in this diagnosis, is problematic 
and frequently results in unnecessary surgical treatment. Using proposed standard morphologic criteria, we conducted a 10-year retrospective review of thyroid FNAs previously categorized as "follicular lesion of undetermined significance" with surgical follow-up to assess whether the indeterminate interpretations could be minimized and cytomorphologic features predictive for neoplasia could be identified.

\section{Materials and Methods}

Between January 1998 and December 2007, our institution performed a total of 3,809 thyroid FNAs among which $220(5.8 \%)$ aspirates were categorized as "follicular lesion of undetermined significance." A SNOMED search was performed in the electronic pathology database and retrieved a total of 123 aspirates in which the indeterminate interpretation was rendered and hemithyroidectomies or total thyroidectomies were subsequently performed. Routine cytomorphologic examination of thyroid FNAs consisted of analysis of air-dried Diff-Quik smears, alcohol-fixed Papanicolaou smears, and ThinPrep and/or cell block preparations. The indeterminate diagnoses were originally rendered as the cytopathologists initially determined that the cytomorphologic features were equivocal and insufficient for definitive classification into either non-neoplastic or neoplastic categories. Suboptimal specimens due to insufficient cellularity, poor preservation, and/or obscuring blood were not categorized into this particular group.

Proposed standard morphologic criteria was established according to Kini's description ${ }^{10}$ with minor modifications. Prior to the retrospective review, images illustrating the standard morphologic criteria with explanations were circulated among the reviewers and an agreement was achieved on the morphologic criteria for cytologic interpretation. Four cytopathologists (X.J., M.H.R., S.M.K., and C.W.M.) participated in the retrospective review. Reviewers' experience ranged from junior to senior level. Subsets of 123 aspirates were randomly distributed among the reviewers who were blinded to corresponding histologic findings at the time of retrospective assessment. Each reviewer performed an independent assessment and a total of 24 individual cytologic features (Table I and Fig. 1) were semi-quantitatively assessed for each aspirate with a score of 0 (absent), 1 (noticeable), 2 (easily seen), and 3 (prominent). Using the institutional eight thyroid FNA diagnostic categories ${ }^{11}$ as a guideline, the reviewers recorded their interpretation on completion of assessment.

Cyto-histologic correlation was performed between the diagnoses rendered at the retrospective review and the corresponding histologic diagnoses. Furthermore, statistical analysis was performed using the logistic regression model to identify cytomorphologic features predictive of histologic diagnosis of neoplasia (both adenoma and carcinoma). A $P$ value of less than 0.05 was considered statistically significant.
Table I. Cytomorphologic Features Assessed During the Retrospective Review

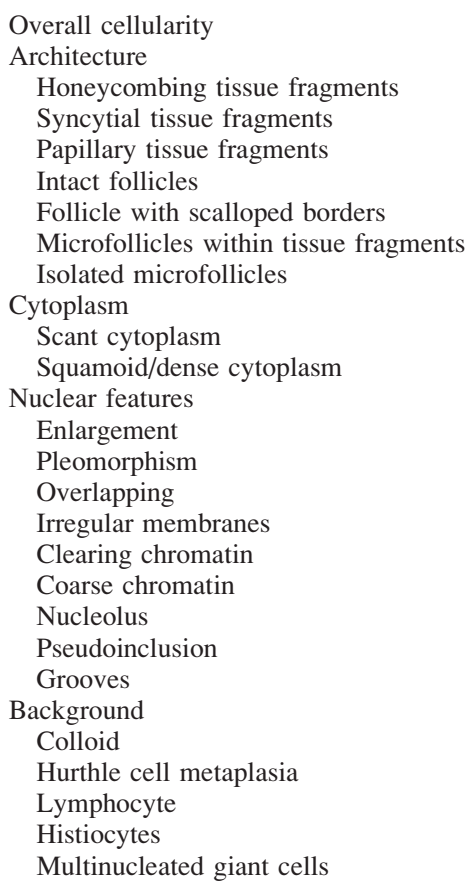

\section{Results}

The retrospective review of a total of 123 thyroid aspirates previously diagnosed as "follicular lesion of undetermined significance" using proposed standard morphologic criteria reclassified $64(52 \%)$ aspirates as non-neoplastic lesions (nodular hyperplasia or chronic lymphocytic thyroiditis) and $27(22 \%)$ aspirates as neoplasms. Of these 27 neoplasms, 22 were reclassified as "follicular neoplasm" and five as "positive for papillary thyroid carcinoma." However, the indeterminate diagnosis remained unchanged in the remaining $32(26 \%)$ aspirates. Table II shows correlation between the cytologic diagnoses rendered by the retrospective review and corresponding histologic diagnoses. Among the 64 aspirates reclassified as non-neoplastic, $47(73.4 \%)$ were confirmed as benign thyroid nodules on corresponding surgical specimens. Of 27 aspirates reclassified as neoplastic, $15(55.6 \%)$ were confirmed as neoplastic by histologic examination (Fig. 2) including nine follicular adenomas, one follicular carcinoma, and five papillary thyroid carcinomas. The diagnostic accuracy reached $68.1 \%(62 / 91)$.

Of 32 aspirates in which the indeterminate interpretation remained unchanged, corresponding histologic examination identified 21 non-neoplastic lesions and 11 neoplasms, including nine follicular adenomas, one follicular carcinoma, and one papillary thyroid carcinoma. Malignancy was thus demonstrated in $6 \%$ of aspirates that remained as indeterminate on the retrospective review. 

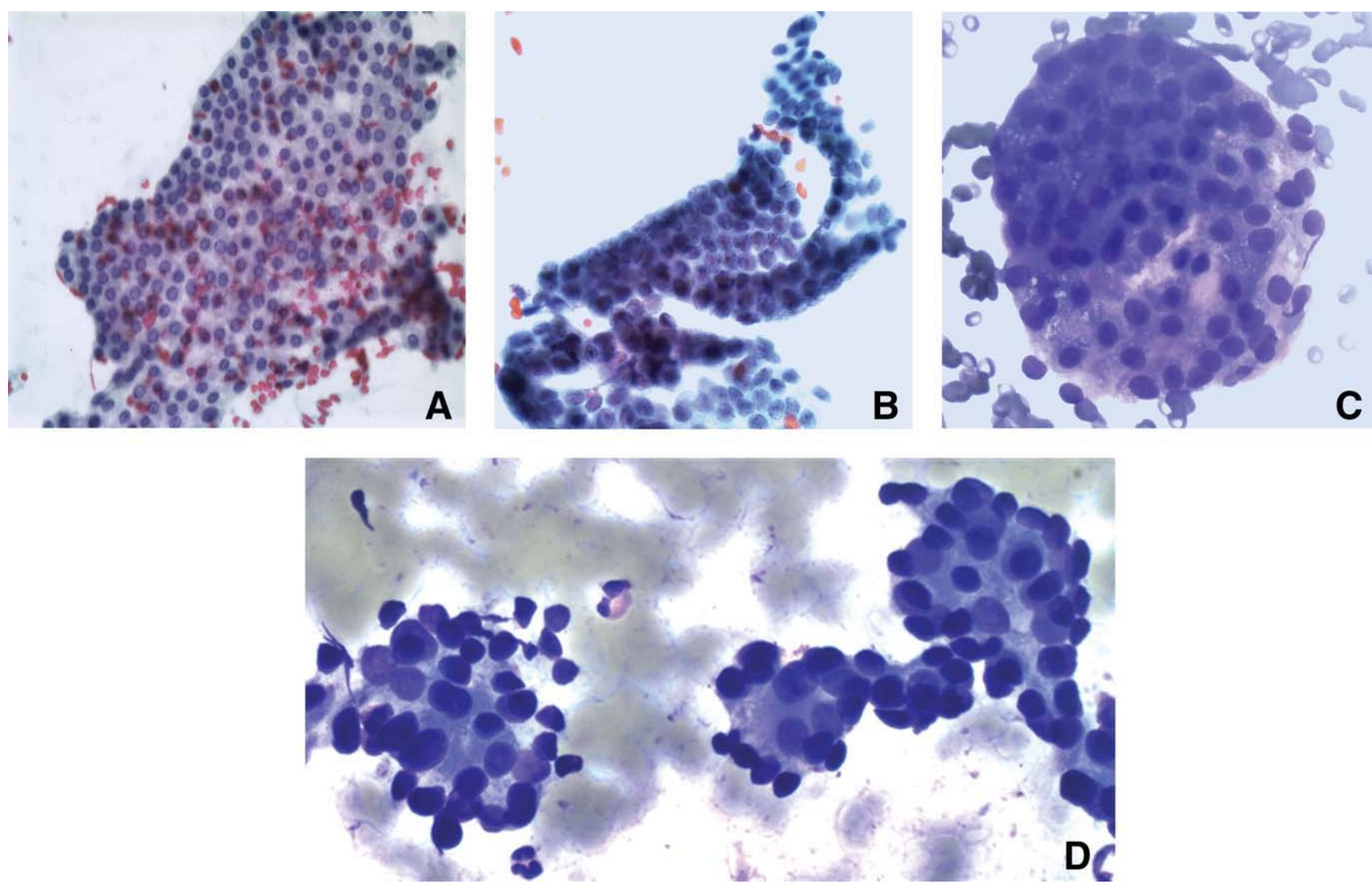

Fig. 1. Example of some cytomorphologic features assessed during the retrospective review. A: The honeycombing tissue fragment showing monolayer sheet of cells with well-defined cytoplasmic borders and evenly distributed nuclei. Nuclear polarity is maintained (Papanicolaou, $\times 600)$. B: Syncytial tissue fragments with cells that have poorly defined cytoplasmic borders. Nuclei are not evenly distributed. Nuclear crowding and overlapping is appreciated. Nuclear polarity is altered (Papanicolaou, $\times 600$ ). C: This intact follicle shows a group of cells forming a sphere. The cells are arranged in a honeycombing pattern (Diff-Quik, ×600). D: Microfollicles. Cells comprising the small follicles show nuclear crowding and overlapping (Diff-Quik, $\times 600$ ). [Color figure can be viewed in the online issue, which is available at wileyonlinelibrary.com.]

Table II. Correlation of Results of Retrospective Cytologic Review and Corresponding Histologic Diagnosis

\begin{tabular}{lcccccc}
\hline & \multicolumn{5}{c}{ Histologic Diagnosis } \\
\cline { 2 - 5 } Cytologic diagnosis & Non-neoplasia & Follicular adenoma & Follicular carcinoma & Papillary carcinoma & Parathyroid adenoma & Total \\
\hline Non-neoplasia & 47 & 9 & 5 & 2 & 1 & $64(52 \%)$ \\
Indeterminate & 21 & 9 & 1 & 1 & - & $32(26 \%)$ \\
Follicular neoplasm & 9 & 9 & 1 & 3 & - & $22(17.9 \%)$ \\
Papillary carcinoma & 3 & -7 & 7 & 8 & $5(4.1)$ \\
Total & 80 & 27 & & & $123(100 \%)$ \\
\hline
\end{tabular}

Table III shows cytomorphologic features that were significantly predictive for histologic diagnosis of neoplasia, along with odds ratios (95\% confidential interval) and $P$ values for each. As demonstrated by the logistic regression model, the histologic diagnosis of neoplasia positively correlated with the presence of the following cytomorphologic features: syncytial tissue fragments; isolated microfollicles; follicles with scalloped borders; scant cytoplasm; irregular nuclear membranes; nuclear overlapping; and coarse chromatin. Increased cellularity was a marginally significant feature that correlated with neoplasia. On the contrary, the presence of follicular cells displaying a hon- eycombed pattern, abundant colloid, and histiocytes inversely correlated with neoplasia in a statistically significant manner.

\section{Discussion}

The committee of the recent NCI Thyroid FNA State of Science Conference defined "follicular lesion of undetermined significance" as a heterogeneous category consisting predominantly of cases in which the cytomorphologic findings are equivocal and neither convincingly demonstrate features justifying a definitive interpretation of a benign thyroid nodule nor demonstrate the degree of cellular/ 

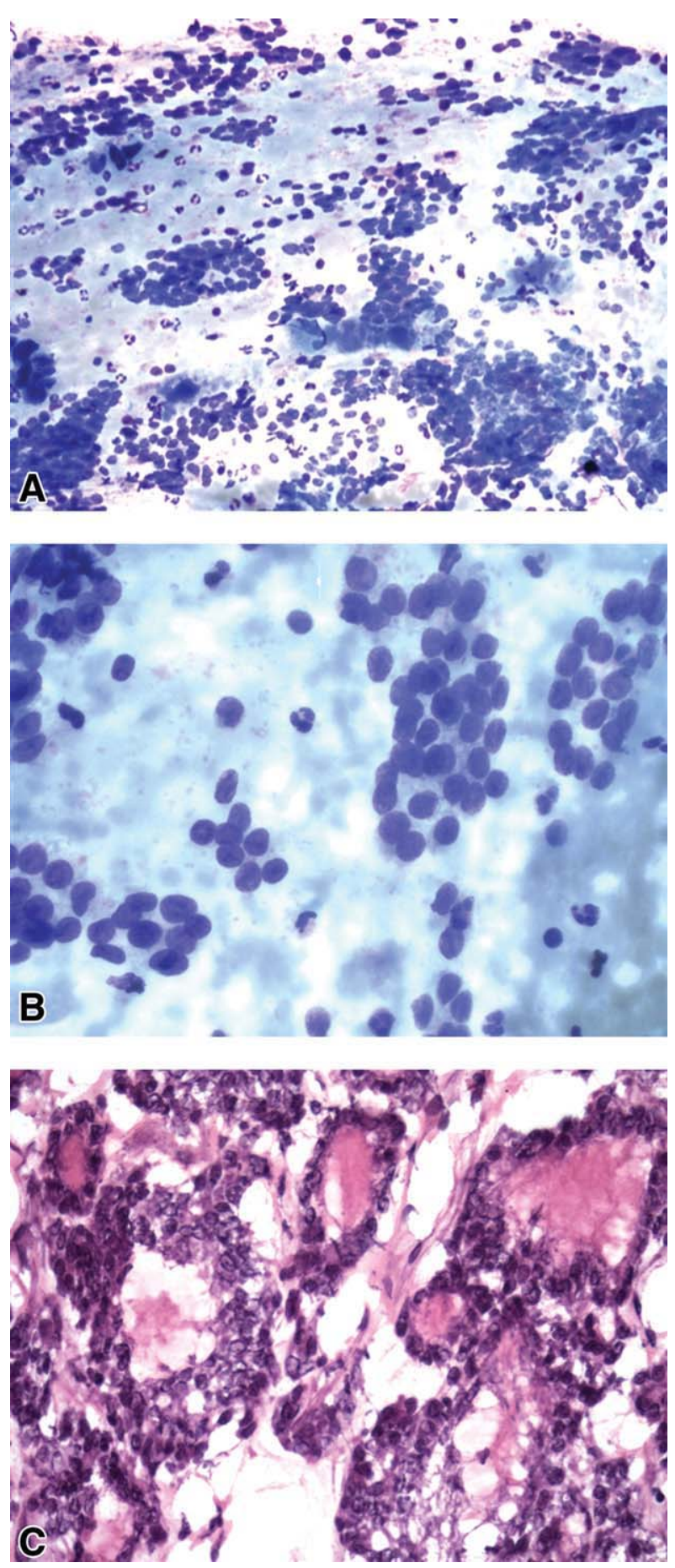

Fig. 2. Conventional smear showing A: hypercellularity with numerous microfollicles (Diff-Quik, $\times 200$ ); B: the follicular cells exhibits nuclear crowding/overlapping, scant cytoplasm, nuclear enlargement with irregular nuclear membranes. No definitive intranuclear grooves and pseudoinclusions are appreciated (Diff-Quik, $\times 600$ ). It was interpreted as follicular neoplasm on the retrospective review. C: The corresponding histology (C) revealing follicular variant of papillary thyroid carcinoma $(\mathrm{H} \& \mathrm{E}, \times 400)$. [Color figure can be viewed in the online issue, which is available at wileyonlinelibrary.com.]
Table III. Predictive Cytomorphologic Features for Neoplasia

\begin{tabular}{lcc}
\hline Features & $\begin{array}{c}\text { Odds ratio } \\
\text { (95\% confidential interval) }\end{array}$ & $P$ value \\
\hline Syncytial tissue fragments & $1.64(1.11,2.42)$ & 0.01 \\
Isolated microfollicles & $1.82(1.20,2.75)$ & 0.005 \\
Follicles with scalloped borders & $1.78(1.02,3.10)$ & 0.04 \\
Scant cytoplasm & $2.08(1.26,3.44)$ & 0.004 \\
Irregular nuclear membranes & $1.69(1.00,2.84)$ & 0.05 \\
Nuclear overlapping & $1.47(1.01,2.16)$ & 0.04 \\
Coarse chromatin & $2.56(1.34,4.97)$ & 0.004 \\
Honeycombing tissue fragments & $0.59(0.41,0.85)$ & 0.004 \\
Colloid & $0.49(0.29,0.81)$ & 0.005 \\
Histiocytes & $0.57(0.35,0.94)$ & 0.03 \\
\hline
\end{tabular}

architectural atypia sufficient for a diagnosis of "follicular neoplasm" or "suspicious for malignancy." The committee proposed that, ideally, the classification of a thyroid FNA as indeterminate be used in less than $7 \%$ of cases. ${ }^{8}$ However, a multi-institutional analysis has demonstrated marked variations in the use of this indeterminate diagnosis among both pathologists $(2.5-28.6 \%)$ and institutions $(3.3-14.9 \%) .^{12}$ Thyroid aspirates carrying the indeterminate interpretation comprised $\sim 6 \%$ of all thyroid FNAs performed in our institution during the 10-year period of the retrospective review, thereby falling within the recommended range.

The marked variations in utilization of the indeterminate diagnosis are thought to be the consequence of the lack of precisely defined, objective morphological criteria. ${ }^{9,12,13}$ Despite this fact, this interpretation should not be used too liberally. Recognition of various architectural patterns and cytologic features is essential in the cytomorphologic evaluation of thyroid FNAs. For example, a small fragment of follicular cells exhibiting evenly spaced, nonoverlapping nuclei should not be misinterpreted as a microfollicle simply because of its small size. There are also concerns related to threshold and level of stringency in applying the diagnostic criteria that may vary among institutions and observers. According to our institutional experience, the presence of a minor population of microfollicles in the setting of predominantly honeycombing tissue fragments, consistent with nodular hyperplasia, does not necessarily upgrade the interpretation to "follicular lesion of undetermined significance" or "suspicious for follicular neoplasm."

There are limited studies on the subclassification of the indeterminate category. ${ }^{13,14}$ Our retrospective review of thyroid FNAs previously categorized as "follicular lesion of undetermined significance" using proposed standard morphologic criteria significantly reduced the number of indeterminate diagnoses from 123 to 32 . The corresponding histologic diagnoses were concordant with the cytologic interpretation in nearly $75 \%$ of the non-neoplastic and more than one half of the neoplastic lesions that were reclassified by the retrospective review. A diagnostic accuracy of nearly $70 \%$ was achieved. Although surgery could have been avoided for nearly three-fourth of the non-neoplastic 
lesions, the review failed to identify 16 neoplastic lesions of thyroid (false-negative). It was noted that the majority of the false-negative cases presented as a solitary nodule. The review also yielded false-positive rate of $20 \%$, which is slightly higher than the previously reported false-positive rate $(16 \%)$. The latter was generated from a study that examined thyroid nodules with definitive cytologic diagnosis of non-neoplasia and neoplasia/malignancy. ${ }^{15}$ Although the diagnostic accuracy of the review appears to be less accurate than previously published data, ${ }^{15,16}$ the differences were anticipated as the current study focuses on difficult thyroid lesions that exhibited equivocal features and thus cause diagnostic challenges. Whether the combination of clinical and cytologic examination is the best approach for selecting surgical candidates has yet to be investigated. ${ }^{13}$

Does the level of a pathologist's experience play a role in the frequency in which thyroid FNAs are interpreted as indeterminate? The results of a retrospective study of 21 indeterminate follicular lesions by Clary et al. ${ }^{13}$ found that increased experience is related to use of more definitive diagnostic categories when a standardized answer form was provided. On the other hand, a multi-institutional study failed to show a relationship between the level of experience and usage of the indeterminate diagnostic category. ${ }^{12}$ The results of the latter study were generated from review of original cytology reports. On the contrary, the results of the former study reported by Clary et al. were generated from a second review of previously diagnosed indeterminate lesions using a standardized answer form with four diagnostic categories (benign/favor benign, follicular lesion, favor neoplastic, and follicular neoplasm) and thus, selection bias existed.

In the current study, the morphologic features that are commonly encountered in cytologic samples and routinely assessed in an appropriate context were evaluated. Prior to the retrospective review, the reviewers discussed images illustrating the standard morphologic criteria and reached an agreement on the morphologic criteria for cytologic interpretation. We hoped that the approach for establishing uniformity among the reviewers would minimize both intraobserver and interobserver variability. Comprehensive assessment of interobserver variability is beyond the scope of the current study. A separate study is being conducted to evaluate interobserver agreement. However, the results of the current study provide a basis for the implementation of routine consensual evaluation of thyroid nodules categorized as "follicular lesion of undetermined significance" in cytology practice. We anticipate that the refined consensual approach will bring enhanced accuracy in the categorization of these difficult thyroid lesions.

Despite the efforts, there are certain lesions that fall into the indeterminate category due to intrinsic features of thyroid nodules and sampling variation, causing diagnostic difficulties during routine cytomorphologic examination. In our study, the interpretation of "follicular lesion of undetermined significance" remained unchanged in 32 aspirates after the retrospective rereview. The corresponding histologic examination revealed one follicular carcinoma and one papillary thyroid carcinoma. This low incidence of malignancy $(6 \%)$ in our study is consistent with the risk of malignancy $(5-10 \%)$ presented at NCI Thyroid FNA State of the Science Conference. ${ }^{8}$ Because of the relatively low-predictive value of malignancy, patients in which this indeterminate cytologic interpretation is rendered on the initial thyroid FNA can benefit from repeat FNA and correlation with clinical and radiologic findings. ${ }^{8}$ Several studies have shown that repeat aspiration yielded a definitive diagnosis for nodules initially diagnosed as indeterminate. ${ }^{17-19}$ Lately, the potential of using molecular analysis as an adjunct of cytology to further refine an indeterminate interpretation of thyroid FNAs has been a topic of discussion. ${ }^{20,21}$

In summary, using proposed standard morphological criteria can help minimize the diagnosis of "follicular lesion of undetermined significance," allow for more accurate cyto-histologic correlation and thereby play a substantial role in reducing unnecessary surgical intervention.

\section{References}

1. Gharib H. Fine-needle aspiration biopsy of thyroid nodules: Advantages, limitations, and effect. Mayo Clin Proc 1994;69:4449.

2. Holleman F, Hoekstra JB, Ruitenberg HM. Evaluation of fine needle aspiration (FNA) cytology in the diagnosis of thyroid nodules. Cytopathology 1995;6:168-175.

3. Mandreker SR, Nadkarni NS, Pinto RG, Menezes S. Role of fine needle aspiration cytology as the initial modality in the investigation of thyroid lesions. Acta Cytol 1995;39:898-904.

4. Zagorianakou P, Malamou-Mitsi V, Zagorianakou N, Stefanou D, Tsatsoulis A, Agnantis NJ. The role of fine-needle aspiration biopsy in the management of patients with thyroid nodules. In Vivo 2005; 19:605-609.

5. Busseniers AE, Oertel YC. Cellular adenomatoid nodules of the thyroid: review of 219 fine-needle aspirates. Diagn Cytopathol 1993;9: 581-589.

6. Poller DN, Ibrahim AK, Cummings MH, Mikel JJ, Boote D, Perry M. Fine-needle aspiration of the thyroid. Cancer 2000;90:239-244.

7. Greaves TS, Olvera M, Florentine BD, et al. Follicular lesions of thyroid: A 5-year fine-needle aspiration experience. Cancer 2000; 90:335-341.

8. Baloch ZW, Cibas ES, Clark DP, et al. The National Cancer Institute Thyroid fine needle aspiration state of the science conference: A summation. CytoJournal 2008;5:6.

9. Shi Y, Ding X, Klein M, et al. Thyroid fine-needle aspiration with atypia of undetermined significance: A necessary or optional category? Cancer Cytopathol 2009;117:298-304.

10. Kini SR. Thyroid cytopathology: An atlas and text. Philadelphia: Lippincott Williams \& Wilkins; 2008. 513 p.

11. Jing X, Michael CW, Pu RT. The clinical and diagnostic impact of using standard criteria of adequacy assessment and diagnostic terminology on thyroid nodule fine needle aspiration. Diagn Cytopathol 2008;36:161-166.

12. Layfield LJ, Morton MJ, Cramer HM, Hirschowitz S. Implications of the proposed thyroid fine-needle aspiration category of "follicular 


\section{JING ET AL.}

lesion of undetermined significance": A five-year multi-institutional analysis. Diagn Cytopathol 2009;37:710-714.

13. Clary KM, Condel JL, Liu Y, Johnson DR, Grzybicki DM, Raab SS. Interobserver variability in the fine needle aspiration biopsy diagnosis of follicular lesions of the thyroid gland. Acta Cytol 2005; 49:378-382.

14. Kini SR, Miller JM, Hamburger JI, Smith-Purslow MJ. Cytopathology of follicular lesions of the thyroid gland. Diagn Cytopathol 1985;1: 123-132.

15. Baloch ZW, Sack MJ, Yu GH, Livolsi VA, Gupta PK. Fine-needle aspiration of thyroid: An institutional experience. Thyroid 1998;8:565-569.

16. Caraway NP, Sneige N, Samaan NA. Diagnostic pitfalls in thyroid fine-needle aspiration: a review of 394 cases. Diagn Cytopathol 1993;9:345-350.
17. Baloch Z, LiVolsi VA, Jain P, et al. Role of repeat fine-needle aspiration biopsy (FNAB) in the management of thyroid nodules. Diagn Cytopathol 2003;29:203-206.

18. Yang J, Schnadig V, Logrono R, Wasserman PG. Fine-needle aspiration of thyroid nodules: A study of 4703 patients with histologic and clinical correlations. Cancer 2007;111:306-315.

19. Yassa L, Cibas ES, Benson CB, et al. Long-term assessment of a multidisciplinary approach to thyroid nodule diagnostic evaluation. Cancer 2007;111:508-516.

20. Nikiforov YE, Steward DL, Robinson-Smith TM, et al. Molecular testing for mutations in improving the fine-needle aspiration diagnosis of thyroid nodules. J Clin Endocrinol Metab 2009;94:2092-2098.

21. Nikiforova MN, Nikiforov YE. Molecular diagnostics and predictors in thyroid cancer. Thyroid 2009;19:1351-1361. 